\title{
Ethanol and avoidance-avoidance conflict in the rat
}

\author{
JAMES G. MANSFIELD, NEWELL K. EATON, CHRISTOPHER L. CUNNINGHAM, \\ and JUDSON S. BROWN \\ University of Oregon Health Sciences Center, Portland, Oregon 97201
}

\begin{abstract}
The effects of ethanol on avoidance-avoidance conflict behavior in a straight runway were studied in rats with a 2 by 2 design in which shock-escape training and subsequent shock-free conflict tests were administered after the animals had orally ingested either ethanol or sugar water. Rats trained under ethanol escaped shock more slowly and, during the conflict tests, approached the shock regions less closely, started and ran more slowly, and oscillated less than sugar-water-trained controls. However, rats tested under ethanol approached the shock regions more closely, started and ran more rapidly, oscillated more, and reversed direction more than rats tested when sober. No state-dependent interactions were obtained. Some of the results were consistent wich a fear-reduction hypothesis, but others supported the assumption that ethanol affected the two avoidance gradients differentially.
\end{abstract}

The view that ethanol significantly reduces anxiety or tension, thereby strengthening acts preceding its ingestion and increasing the likelihood of abuse, still has adherents. Cappell and Herman (1972), however, have noted that empirical evidence to support this "tension-reduction" hypothesis is scanty. In only one area, that of conflict behavior, has it been upheld with any reasonable degree of consistency. Early examples are provided by the work of Conger (1951, 1956) and Masserman and Yum (1946) and, more recently, in experiments by Freed $(1967,1968)$. All of these investigators have concluded that alcohol tends to bring about closer approach to a goal region where both rewards and punishments have previously been administered. Typically, such effects have been interpreted as indicating that ethanol selectively weakens avoidance relative to approach by attenuating conditioned fear.

The purpose of the present experiment was to determine how ethanol affects behavior in an avoidance-avoidance conflict situation and, in particular, to determine whether it tends, presumably by simultaneously weakening both of the competing avoidance tendencies, to increase the likelihood that both aversive regions will be approached more closely.

The avoidance-avoidance paradigm offers several possible advantages over the approach-avoidance

Supported in part by a predoctoral fellowship to C. L. Cunningham from the National Institute of General Medical Studies, by Training Grant AA07074 and Research Grant AA01229 from the National Institute of Alcohol Abuse and Alcoholism, and by Research Grant MH23607 from the National Institutes of Health. The second author is now at U.S. Army Research Institute, Fort Knox, Kentucky. The third author is now at Yale University, New Haven, Connecticut. A. J. Skurdal's critique of the manuscript is acknowledged with thanks. design employed hitherto. First, only one putative source of motivation (conditioned fear) is involved rather than two (hunger and fear). Hence, any effects of ethanol cannot be ascribed to some complex, undetected, fear-hunger interaction. Second, in the approach-avoidance procedure, if ethanol is accompanied by closer goal approach, a tension-fear reduction interpretation can be supported only if it can be shown that the strength of the approach was not affected by the drug. Conger (1951) provided such evidence, but few others have. Third, with the avoidance-avoidance procedure, the two competing tendencies can be built up simultaneously by means of alternating shock-escape trials from each end of an alley. In the approach-avoidance situation, however, the approach habit must be established first, since animals cannot readily be persuaded to approach a region for food after they have been punished there. As Barry and Miller (1962) have noted, under these conditions, it is difficult to distinguish a drug that reduces fear from one which merely affects a more recently learned habit.

Theoretical predictions as to the effects of ethanol on avoidance-avoidance conflicts have recently been outlined by Brown and Crowell (1974) in their extension of Miller's (1944, 1959) conflict theory. According to their analysis, aversive stimulation (electric shock) administered at each end of a straight runway strengthens tendencies to avoid both locations. Moreover, these tendencies (on nonshock trials) are assumed to be maximally strong at the place where the shock was administered and to decrease with distance therefrom. The heavy dashed lines of Figure 1 represent such hypothetical gradients. On the further assumption that the effective tendency to move in either direction is 
directly related to the net (algebraic) difference between the oppositely directed tendencies at every point, it follows that, no matter where the animal is placed, it should move toward the intersection $\left(I_{2}\right)$ of the gradients where the net tendency is zero. Clearly, the tendency to move away from a shock zone will be stronger the closer the animal is to that zone, since the difference between the left- and right-going tendencies increases with nearness to such an area.

An additional assumption made by Brown and Crowell was that such gradients will fluctuate rather widely (independently of one another) from moment to moment due to uncontrolled changes in the total internal and external stimulus complex. In Figure 1, the lighter dashed lines above and below the heavier lines represent fictitious upper and lower limits of such fluctuations. A major result of such changes is that the intersections of the gradients will shift back and forth between points $I_{1}$ and $I_{3}$. These points thus delimit both the range of oscillatory movements the animal will make and how closely each shock region will be approached.

If fear potentiates both locomotor tendencies and if alcohol reduces fear, then the heights and slopes of both gradients should be simultaneously and equally reduced by alcohol and the distance between the extreme positions of the intersections $\left(I_{1}\right.$ and $\left.I_{3}\right)$ should be increased. Behaviorally, this should be reflected in an expansion in the width of the range of the animal's oscillatory movements and in closer approaches to each punishment region. With lowered gradients, one would also expect slower running speeds throughout the alley and slower starting speeds, because the difference between the opposing gradients would be diminished at all points.

To test these predictions, rats were first given several shock-escape training trials on which they were taught to run away from each end of a straight runway. On these trials, a compound $\mathrm{CS}$, consisting of a light and a tone, was presented at the end from which the escape trial was initiated. Avoidanceavoidance conflict test trials were conducted without shock and with two light-tone CSs present, one at each end of the runway. Continuous quantitative records were obtained throughout the test period of speed of locomotion and of position in the alley. In the 2 by 2 factorial design, half the rats were trained after they had drunk an alcohol solution and half after they had ingested sugar water. Half the members of each of these groups were tested when sober and half when inebriated.

\section{METHOD}

\section{Subjects}

The subjects were 40 naive 130-day-old Sprague-Dawley-derived female albino rats from Carworth Farms, Inc., Portage, Michigan, initially weighing $240-260 \mathrm{~g}$. They were maintained in individual

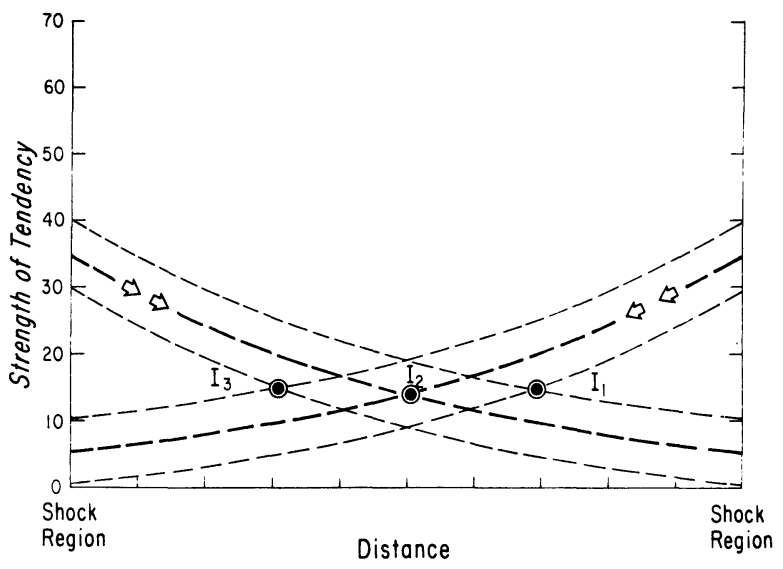

Figure 1. Two avoidance gradients (heavy dashed lines) resulting from the administration of punishment at each of two spatially separated regions. The expected range of left-right oscillatory movement lies between the intersections $I_{1}$ and $I_{3}$ defined by maximum upward and downward fluctuations (light dashed lines) in the strengths of the tendencies.

cages throughout the study in a room with a 12-h light/dark cycle. Purina Lab Chow was available ad lib at all times, but the rats were allowed access to fluid (water, sugar water, or sugar water and ethanol) for only one 15-min period each day. This period began approximately $3 \mathrm{~h}$ before the end of the light cycle, and the experimental treatments followed this period. The experiment was conducted in two replications, with 20 rats in each replication.

\section{Apparatus}

A wooden, grid-floored, medium-gray alley $(184 \mathrm{~cm}$ long, $8.7 \mathrm{~cm}$ wide, $18.5 \mathrm{~cm}$ deep) was used for all training and testing trials. A translucent, ground-glass panel $(8.7 \times 7.5 \mathrm{~cm})$ was inset into each end wall and the entire lid was constructed of clear Plexiglas. To minimize handling cues, a trap-door-floored starting box $(19.5 \mathrm{~cm}$ long, $7.3 \mathrm{~cm}$ wide, $11 \mathrm{~cm}$ deep, with a translucent Plexiglas lid) was employed which could be positioned above the alley at any desired point. The grid floor, with its 2.4-mm-diam stainless steel bars spaced at 2.75 -cm intervals, was supported independently of the alley on two cantilever arms, one at each end. Strain gauges cemented to these arms were arranged in a Wheatstone-bridge configuration in such a way that the output voltage reflected the rat's position in the alley with an accuracy, for the rat's center of mass, of $\pm 4 \mathrm{~cm}$ over the range of $184 \mathrm{~cm}$. This voltage was measured every $.20 \mathrm{sec}$ by a digital voltmeter (Hewlett-Packard 3550A) and was recorded by a punched-paper tape system. The tapes were subsequently read and analyzed by means of a PDP/8F mini-computer. The CS was a compound light-tone stimulus that was turned on and off at a rate corresponding to a $2-\mathrm{Hz}$ square wave. The light component was provided by two $28-\mathrm{V}$ clear miniature lamps (Chicago Miniature, CM1819) one at each end of the alley, positioned $2 \mathrm{~cm}$ outside the ground-glass diffusing panels. The tone was generated by two Sonalert signaling devices $(4.5 \mathrm{kHz}$, Mallory, SC628H), one being mounted $16 \mathrm{~cm}$ beyond each end of the alley. The individual intensities of the two Sonalert signals were adjusted to a level of $80 \mathrm{~dB}$, re $.0002 \mathrm{dyne} / \mathrm{cm}^{2}$, as measured by a Scott noise-level meter (Model 450B) placed inside the alley $13 \mathrm{~cm}$ from the end.

\section{Procedure}

Table 1 summarizes the major treatments administered to the four groups in the 2 by 2 design. Groups A-A and A-SW were given shock escape training after ingesting an alcohol solution 
Table 1

Summary of Treatments Imposed On, and Solutions Drunk By, the Four Experimental Groups

\begin{tabular}{rlclll} 
& & & \multicolumn{2}{c}{ Iluids Drunk } \\
Day & Treatment & $\begin{array}{c}\text { Group } \\
\text { A-A }\end{array}$ & $\begin{array}{c}\text { Group } \\
\text { SW-SW }\end{array}$ & $\begin{array}{c}\text { Group } \\
\text { A-SW }\end{array}$ & $\begin{array}{l}\text { Group } \\
\text { SW-A }\end{array}$ \\
\hline $1-3$ & Handling & Water & Water & Water & Water \\
4 & Handling & SW & A & SW & A \\
5 & Handling & A & SW & A & SW \\
6 & Handling & SW & A & SW & A \\
7 & Habituation & A & SW & A & SW \\
8 & Fluid Only & SW & A & SW & A \\
9 & Habituation & A & SW & A & SW \\
10 & Fluid Only & SW & A & SW & A \\
11 & Training 1 & A & SW & A & SW \\
12 & Fluid Only & SW & A & SW & A \\
13 & Training 2 & A & SW & A & SW \\
14 & Fluid Only & SW & A & SW & A \\
15 & Training 3 & A & SW & A & SW \\
16 & Fluid Only & SW & A & SW & A \\
17 & Training 4 & A & SW & A & SW \\
18 & Testing 1 & A & SW & SW & A \\
19 & Fluid Only & SW & A & A & SW \\
20 & Testing 2 & A & SW & SW & A \\
\hline
\end{tabular}

Note $-A=$ alcohol plus sugar water, $S W=$ sugar water .

(A), while Groups SW-SW and SW-A were trained after having drunk sugar water (SW). Groups A-A and SW-A received shock-free conflict tests (Days 18 and 20) after consuming alcohol, while the remaining groups were tested after drinking sugar water. The sugar water solution consisted of roomtemperature tap water plus granulated cane sugar $(80 \mathrm{~g} /$ liter $)$. Two alcohol solutions were used: one contained $3.17 \%$ ethanol, and the other $4.75 \%$ ethanol, both with the same concentration of sugar as in the sugar-water solution. The sugar was added to mask the unpleasant taste of ethanol (Eckhardt, 1974) and to increase the amount of fluid consumed. The $3.17 \%$ solution was administered on Days 4-7, and the $4.75 \%$ solution was used on all remaining experimental days (all concentrations $\mathrm{v} / \mathrm{v}$ ).

On each of the first 6 days, the animals were handled individually for $1 \mathrm{~min}$, during which time they were also weighed. Then they were permitted to drink the scheduled solution for $15 \mathrm{~min}$, after which they were again handled for $1 \mathrm{~min}$. The habituation experiences on Days 7 and 9 consisted of a 2 -min period to explore the apparatus. On the "fluid only" days, the rats were allowed to drink whichever of the two solutions they had not drunk while being trained. This procedure guaranteed that all animals had equal experiences with both solutions and with day-to-day shifts from one to the other.

Days $11,13,15$, and 17 were training days on which every rat received two shock-escape trials, one from each end of the alley. At the beginning of the trial, the rat was placed in the starting box. As soon as the rat faced the nearest end of the alley, the tonelight CS was turned on, and 1 sec later the rat was dropped onto the electrified grid. After the animal had escaped the shock and had run past the midpoint of the alley, or after the CS had been on for $15 \mathrm{sec}$ (whichever came first), the CS was terminated and the rat was returned to its carrying box in the experimental room. The animals were run in rotation so that their training trials were well distributed, the intertrial interval for each day averaging 25 to $30 \mathrm{~min}$. Over the 4 training days, each rat received a toial of eight escape trials, four from each end of the alley. On the first day, a shock of $55 \mathrm{~V}(\mathrm{ac}, 60 \mathrm{~Hz})$ was applied through a $10 \mathrm{k} \Omega$ series resistor to the $30 \mathrm{~cm}$ of the grid at the end where the rat was dropped. On the 3 subsequent training days, the shock was set at 60,60 , and $65 \mathrm{~V}$, respectively.

Conflict trials (two per day) were administered on Days 18 and
20 to two groups (A-A and SW-A) after they had ingested alcohol. The other two groups (A-SW and SW-SW) were tested following the drinking of sugar water. Each test trial involved dropping the rat at one of four locations: (a) the left end of the alley, (b) $30 \mathrm{~cm}$ to the left of center, (c) $30 \mathrm{~cm}$ to the right of center, and (d) the right end of the alley. These trials were $30 \mathrm{sec}$ long, and no shock was present, but the intermittent lights and tones were on at both ends of the alley simultaneously. The order of the starting location was varied for each rat, being selected at random from the 24 possible combinations of the four locations. On test trials, as on training trials, the CS was turned on when the rat faced the nearest end of the alley and the rat was dropped onto the grid $1 \mathrm{sec}$ later. For habituation and training days, the order of running was varied systematically; for testing days, the order was varied randomly. Habituation, training, and testing occurred within the hour beginning $30 \mathrm{~min}$ after the drinking period. Earlier work in our laboratory (Eckhardt, 1974), with the same strain of rat under similar conditions, suggests that during this period blood alcohol concentration reaches a peak and remains at a relatively high level.

\section{RESULTS}

\section{Alcohol Dosage}

The mean alcohol dosage for the alcohol-trained groups (A-A and A-SW) averaged over the 4 training days, was $2.22 \mathrm{~g} / \mathrm{kg}$ (range $=.62$ to $3.47 \mathrm{~g} / \mathrm{kg}$ ). Mean dosages for the 2 test days were: Group A-A, $2.37 \mathrm{~g} / \mathrm{kg}$ (range $=1.33$ to $3.59 \mathrm{~g} / \mathrm{kg}$ ); Group SW-A, $2.36 \mathrm{~g} / \mathrm{kg}$ (range $=.99$ to $3.25 \mathrm{~g} / \mathrm{kg}$ ). An analysis of the amounts of fluids consumed revealed that there were no significant differences between groups in ethanol intake during the seven 15-min exposures to alcohol prior to testing.

\section{Starting Speed During Escape Training}

Starting speed was defined as the speed, in $\mathrm{cm} / \mathrm{sec}$, with which the first $30 \mathrm{~cm}$ of the alley was traversed. During acquisition, this segment was electrified. Starting speeds were evaluated by an analysis of variance procedure in which drug treatment (alcohol vs. sugar water) and days of training were factors. Both of these effects proved to be significant, with animals trained under ethanol running more slowly (mean $=22.28 \mathrm{~cm} / \mathrm{sec}$ ) than those trained after consuming sugar water (mean $=30.88 \mathrm{~cm} / \mathrm{sec}$ ) $[\mathrm{F}(1 / 36)=4.32, \mathrm{p}<.05]$. As expected, speeds over days improved with practice $[\mathrm{F}(3 / 108)=9.35$, $\mathrm{p}<.01]$.

\section{Conflict Test Results}

Six different performance measures were obtained from the position data (recorded every $.20 \mathrm{sec}$ ). Group means for each of the six dependent variables are listed in Table 2, and are considered in turn.

Shock-region approach. During each 30 -sec test trial, measurements were made of the closest approach to each of the two ends of the alley where shocks had been administered during training. No measurements were made, however, until after an animal had 
Table 2

Data Obtained on Avoidance-Avoidance Conflict Trials for Groups Trained and Tested Under the Influence of Alcohol and/or Sugar Water

\begin{tabular}{lcccccc}
\hline Groups & $\begin{array}{c}\text { Shock Region } \\
\text { Approach }(\mathrm{cm})\end{array}$ & $\begin{array}{c}\text { Total Oscil- } \\
\text { lation }(\mathrm{cm})\end{array}$ & $\begin{array}{c}\text { Starting Speed } \\
(\mathrm{cm} / \mathrm{sec})\end{array}$ & $\begin{array}{c}\text { Reversals in } \\
\text { Direction }\end{array}$ & $\begin{array}{c}\text { Length of Initial } \\
\text { Run }(\mathrm{cm})\end{array}$ & $\begin{array}{c}\text { Running Speed } \\
(\mathrm{cm} / \mathrm{sec})\end{array}$ \\
\hline SW-A & 29.45 & 568.8 & 30.59 & 3.70 & 145.3 & 33.59 \\
A-A & 51.75 & 482.4 & 18.69 & 3.35 & 105.4 & 27.30 \\
SW-SW & 44.85 & 411.8 & 26.08 & 2.87 & 94.8 & 26.48 \\
A-SW & 73.25 & 345.4 & 13.67 & 2.52 & 49.9 & 16.00 \\
\hline
\end{tabular}

Vote-Row differences reflect the four treatment-test combinations of the 2 by 2 design. All values are means.

made one reversal of direction at least $10 \mathrm{~cm}$ in length. Had this precaution not been observed, endstarting locations would have been spuriously recorded as approaches to the shock region. The two values were averaged and an analysis of variance procedure was applied to the data arranged in a three-factor mixed pattern involving drug during treatment, drug during testing, and starting location (end vs. middle). This analysis yielded a significant main effect of treatment during training $[F(1 / 36)=$ $8.76, p<.01]$ and during testing $[F(1 / 36)=4.64$, $\mathrm{p}<.05$ ], but neither starting location nor any of the interactions of the three factors proved to be significant. When appropriately combined, the group means in the first column of Table 2 indicate that animals tested under the influence of alcohol approached the shock regions more closely than animals tested after drinking sugar water (40.5 vs. $59.05 \mathrm{~cm}$ ) and that alcohol-trained rats approached the ends less closely than sugar-water-trained animals (62.5 vs. $37.15 \mathrm{~cm}$ ).

Total oscillation. Total oscillation was defined as the total amount of movement exhibited during a $30-\mathrm{sec}$ test trial. This measure is independent of shock-region approach, since an animal can accumulate a high oscillation score without approaching either shock region closely or it can approach both regions closely without running up a high score.

As Table 2 shows, the total oscillation scores of animals tested after drinking ethanol were greater than those of rats tested after drinking sugar water $(526.6$ vs. $378.65 \mathrm{~cm})$. Also, training under alcohol yielded lower combined levels than training under sugar water $(413.95$ vs. $490.3 \mathrm{~cm})$. The total oscillation scores were also affected by starting position, with end-dropped animals attaining higher scores than center-dropped rats $(469.75$ vs. $434.42 \mathrm{~cm})$. These latter scores are not listed in Figure 2. All of the main effects were statistically significant $[F(1 / 36)$ $=7.38, \mathrm{p}<.05 ; \mathrm{F}(1 / 36)=27.33, \mathrm{p}<.01 ;$ $F(1 / 36)=7.27, p<.05]$ for training conditions, testing conditions, and starting position, respectively. None of the interactions approached significant levels.

Starting speed. Starting speed was defined, as in shock-escape training, as speed of movement through the first $30 \mathrm{~cm}$. Mean values for the four groups are listed in Column 3 of Table 2. Statistical analysis revealed that starting speeds were slower for alcoholtrained than for sugar-water-trained animals $[F(1 / 36)$ $=4.71, \mathrm{p}<.05]$. None of the other effects was significant.

Reversals in direction of movement. A reversal in direction was scored when a rat turned around (or perhaps backed up) and moved $10 \mathrm{~cm}$ in a direction opposite to that in which it had been traveling. Ten centimeters of continuous movement in one direction at the beginning of a trial was the criterion for establishing direction of travel. Mean numbers of reversals for the four groups are shown in Table 2, where it will be seen that animals tested under ethanol changed their direction of locomotion more frequently than animals tested after consuming sugar water $[F(1 / 36)=9.26, p<.01]$. Neither dropping position nor training condition, nor any interaction, was significant.

Length of initial run. This was stipulated as distance traversed from the starting point to the first pause point. A pause was defined as a period of $2 \mathrm{sec}$ in which the rat did not move forward more than $10 \mathrm{~cm}$. A reversal in direction was counted as a pause if the rat remained within $5 \mathrm{~cm}$ of the turning point, that is, if it did not move a total distance of $10 \mathrm{~cm}$ within a 2-sec period. An overall analysis supported the conclusions that training condition, testing

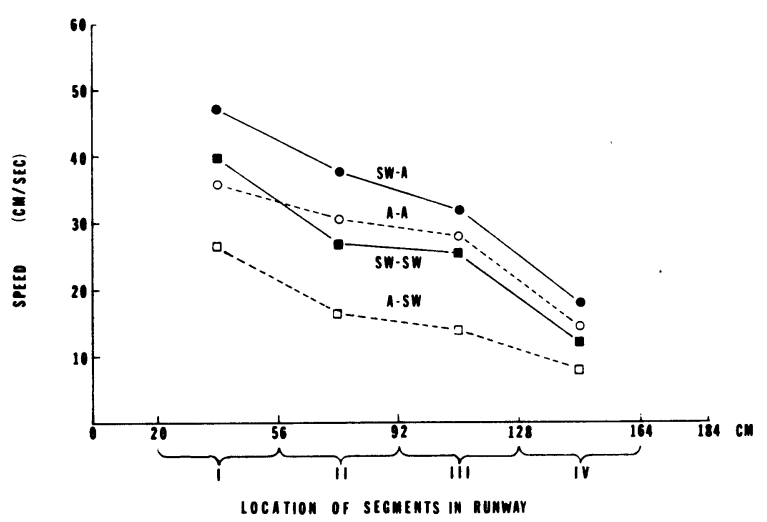

Figure 2. Mean speeds for the initial run through the middle four alley segments. Dashed lines represent speeds for groups trained after drinking ethanol and tested after drinking either ethanol or sugar water. Solid lines represent speeds for groups trained after drinking sugar water, and tested after drinking either ethanol or sugar water. 
condition, and starting location all contributed significantly to the total variance $[\mathrm{F}(1 / 36)=8.64$, $\mathrm{p}<.01 ; \mathrm{F}(1 / 36)=13.56, \mathrm{p}<.01 ; \mathrm{F}(1 / 36)=4.42$, $\mathrm{p}<.05$, respectively]. As the appropriate means in Table 2 show, animals tested under alcohol run farther before pausing than those under sugar water $(125.4$ vs. $72.31 \mathrm{~cm})$ and alcohol-trained rats ran less far than sugar-water-trained animals ( 77.66 vs. $120.05 \mathrm{~cm}$ ). Subjects started at the ends ran farther than those started near the center $(109.6 \mathrm{vs} .57 .1 \mathrm{~cm})$.

Running speed. Running speeds, on only those trials when the animals were started from an end position, were calculated for each subject in each of the four $36-\mathrm{cm}$ segments in the middle $144-\mathrm{cm}$ region of the alley. Speed data from the two $20-\mathrm{cm}$ terminal sections were not included because of the confounding effects of accelerations and decelerations associated with starting and stopping. If a rat stopped, or turned around before reaching any segment, a speed of $0 \mathrm{~cm} / \mathrm{sec}$ was assigned for that segment on that trial. Since 40 rats were each given two end-start test trials, a total of 320 zero scores over four segments was possible. Actual frequencies of zero scores were: $0,1,5$, and 17 , for the $1 \mathrm{st}, 2 \mathrm{nd}, 3 \mathrm{rd}$, and 4 th segments, respectively. If a rat entered a segment but did not complete a run through it, an average speed score for the actual distance traversed was assigned. Mean speeds, averaged over all segments, are listed by groups in Table 2, while means by segments are plotted in Figure 2. It is evident from these data that alcohol-tested rats tended to run faster than animals tested after drinking sugar water. It is also obvious that the speeds for all four groups decreased over segments. (All groups, of course, showed initial accelerations at the start of each run that are not revealed by these segment speeds.) Less immediately apparent is the finding that animals trained under alcohol (dashed lines) ran less rapidly than those trained after drinking sugar water. All of these effects were confirmed as significant by a mixed analysis of variance involving the factors of training, testing, and alley segment $[\mathrm{F}(1 / 36)=7.91$, $\mathrm{p}<.01 ; \mathrm{F}(1 / 36)=9.53, \mathrm{p}<.01 ; \mathrm{F}(3 / 108)=76.27$, $\mathrm{p}<.01$, respectively]. None of the interactions was significant.

An overall examination of the data in Table 2 reveals considerable consistency in that all four groups were rank ordered identically by four of the measures. On the two measures (shock-region approach and starting speeds) for which this did not hold, only the ranks of the two middle groups (A-A and SW-SW) were inverted.

\section{DISCUSSION}

In this experiment, animals given shock-escape training under the influence of ethanol differed from sober controls both on shock-escape training trials and on subsequent shock-free conflict test trials. Drug treatment during training significantly affected five of the six dependent measures recorded during the conflict tests. Specifically, rats trained to escape under ethanol, compared to those trained when sober, tended, on conflict test trials, (a) to approach the shock regions less closely, (b) to exhibit less total back and forth movement, (c) to start more slowly, (d) to run through the middle alley segments less rapidly, and (e) to run less far on the initial run. Alcohol- and sugar-water-trained animals failed to differ only with respect to number of reversals of direction of locomotion.

One interpretation of these training-condition effects rests on the finding that animals trained when inebriated ran significantly more slowly in escaping from shock than did those trained when sober. Regardless of whether this difference was due to an analgesic effect or to motor impairment, it could be argued that the inebriated rats were learning a "slowrunning" habit as compared with the sober animals. Such a micromolar interpretation (Logan, 1956) would fit most of the training-effect findings. Of course, if ethanol had an analgesic effect, the noxiousness of the shock for alcohol-trained rats would have been reduced and fear conditioned to the starting area cues might have been weakened. On the traditional gradient theory (Brown \& Crowell, 1974), if fear is attenuated, the animals should start and run more slowly, the shock region should be approached more closely, the initial run should be longer, and the total movement should be greater. Since only the first two of these outcomes were observed, either the effective intensity of the US was not reduced by alcohol or the gradients were not flattened in the predicted fashion, or both.

The results of drug conditions during conflict trials are of special interest because of their bearing on the theoretical relations under evaluation. During these trials, animals tested after consuming alcohol approached the two ends of the runway more closely, attained higher total oscillation scores, and ran farther on their first run before pausing than did animals tested after drinking sugar water. These outcomes are consistent with the approach-avoidance data previously reported by Conger (1951), Freed (1967), and Masserman and Yum (1946). Conceivably, alcohol reduced the fear that had been conditioned to the cues at the shock regions, lowered and flattened the avoidance gradients (refer to Figure 1), and increased the separation between the maximum positions of the intersections.

Such an interpretation is not consistent, however, with the starting- and running-speed data. If the heights of both gradients had been lowered by ethanol, starting speeds should have been reduced. In actual fact, alcohol-tested rats started faster than those tested under sugar water, though the probability 
associated with that difference $(p=.065)$ failed to reach the conventional value (.05). Moreover, a simultaneous lowering of both gradients would lead, in theory, to slower, rather than faster, speeds in the middle of the alley, an outcome we did not observe.

An explanation of both the speed data and other findings based on the idea that alcohol increases conditioned fear (Amit, Ziskind, \& Baum, 1973) falls short for two reasons. First, alcohol is alleged to be aversive and hence to augment conditioned fear only when it is novel. In this experiment, however, all animals repeatedly experienced both the drug and the sugar-water solutions prior to all training and testing trials. Second, any increase in fear would be expected to elevate the heights and (perhaps) steepen the slopes of both gradients (refer to Figure 1), thereby reducing the average separation of the intersections. The theoretical result would be less close approach to either shock region, shorter initial run, less total oscillation, and so on, outcomes we did not obtain.

A more attractive possibility, which we shall term the "asymmetry hypothesis," is that ethanol affects the two avoidance gradients differentially. For example, our findings are consistent with the assumption that ethanol had a greater weakening effect on the gradient appropriate to the end being approached than on the gradient for the end being avoided. On this view, the response of leaving the area where the trial is initiated is tightly controlled by the nearby salient cues (including the startbox and the stimulation attending being dropped) which have been present repeatedly during training. Hence, that behavior is resistant to modification by moderate doses of ethanol. The tendency to move away from the distant end, when that end is being approached on a conflict trial, is somewhat weaker in comparison, being under the control, at that instant, of remotely seen generalized cues. But that tendency, on a conflict trial, plays the role of a competitive inhibitor which works increasingly to bring the animal to a halt as movement continues. If alcohol weakens inhibitory processes more than it does excitatory ones (Miller \& Barry, 1960), then alcoholtested animals might be expected to approach a threatening region more swiftly and more closely than would sober ones. Perhaps the inebriated animal is less sensitive to the distal stimuli that act to inhibit the instrumental behavior of approaching the opposite end of the runway. Data consistent with the asymmetry hypothesis are provided by studies in which two-way avoidance is facilitated by ethanol (Broadhurst \& Wallgren, 1964; Chesher, 1974; Crow, 1966), in which passive avoidance is impaired by ethanol (Holloway, 1972), in which ethanol appears to degrade fear-inhibitory mechanisms more than fear-excitatory ones (Litner
\& Weisman, cited in Cappell \& Herman, 1972), and in which ethanol facilitates exploration of unfamiliar places (Cutler, Mackintosh, \& Chance, 1975). Additionally, in several unpublished experiments in our laboratory, involving a single avoidance tendency, ethanol has been found to have little effect on the speed with which animals leave the shock region, though it substantially increases the closeness with which that region is approached from the opposite end. Inebriated animals seem to be less able to inhibit tendencies to approach a shock region despite being able to leave that area as fast as do sober animals.

One finding that does not easily fit any of the foregoing interpretations is the increase in number of reversals under ethanol. Perhaps the increased running speeds generate higher numbers of reversals simply because with higher speeds the intersections of the avoidance gradients are reached more quickly. Alternatively, it may be that ethanol augments the rate with which excitatory tendencies fluctuate in strength over time.

Finally, this experiment yielded no evidence whatever of state-dependency effects. Animals trained under ethanol differed, on conflict test trials, from animals trained under sugar water, but none of the Training by Testing interactions was significant. That is, the differences between the ethanol-tested and sugar-water-tested subjects were independent of whether their training had been conducted under ethanol or under sugar water. Conceivably, statedependency effects were obliterated by the repeated shifts for each animal from alcohol to sugar water, and vice versa, during the preliminary habituation period.

\section{REFERENCES}

Amit, Z., Ziskind, D., \& Baum, M. Drug effects and avoidance-extinction in rats: $A$ test of the drug novelty hypothesis using ethanol. Animal Learning \& Behavior. 1973, 1, 41-43.

Barry, H. III, \& Miller, N. E. Effects of drugs on approach-avoidance conflict tested repeatedly by means of a "telescope alley." Journal of Comparative and Physiological Psychology, 1962, 55, 201-210.

Broadhurst, P. L., \& Wallgren, H. Ethanol and the acquisition of a conditioned avoidance response in selected strains of rats. Quarterly Journal of Studies on Alcohol, $1964,25,476-489$.

Brown, J. S., \& Crowell, C. R. Alcohol and conflict resolution: A theoretical analysis. Quarterly Journal of Studies on Alcohol, 1974, 35, 66-85.

Cappell, H., \& Herman, C. P. Alcohol and tension reduction: A review. Quarterly Journal of Studies on Alcohol, 1972, 33. 33-64.

CHesher, G. B. Facilitation of avoidance acquisition in the rat by ethanol and its abolition by $\alpha$-methyl p-tyrosine. Psychopharmacologia, 1974, 39, 87-95.

Conger, J. J. The effects of alcohol on conflict behavior in the albino rat. Quarterly Journal of Studies on Alcohol, 1951, 12. $1-29$.

Conger, J. J. Alcoholism: Theory, problem and challenge. II. 
Reinforcement theory and the dynamics of alcoholism. Quarterly Journal of Studies on Alcohol, 1956, 17, 296-395.

CROw, L. T. Effects of ethanol on conditioned avoidance responding. Physiology and Behavior, 1966, 1, 89-91.

Cutler, M. G., Mackintosh, J. H., \& Chance, M. R. A. Effects of the environment on the behavioral response of mice to nontoxic doses of ethyl alcohol. Neuropharmacology, 1975, 14, 841-845.

ECKARDT, M. J. Conditioned taste aversion produced by the oral ingestion of alcohol in the rat. Doctoral dissertation, University of Oregon Health Sciences Center, Portland, Oregon, 1974.

FREED, E. X. The effect of alcohol upon approach-avoidance conflict in the white rat. Quarterly Journal of Studies on Alcohol, 1967, 28, 236-254.

FREED, E. X. Effect of self-intoxication upon approach-avoidance conflict in the rat. Quarterly Journal of Studies on Alcohol, 1968, 29. 323-329.

Holloway, F. A. State-dependent effects of ethanol on active and passive avoidance learning. Psychopharmacologia, 1972, 25. 238-261.
Logan, F. A. A micromolar approach to behavior theory. Psychological Revie'n', 1956, 53, 63-73.

Masserman, J. H., \& YUM, K. S. An analysis of the influence of alcohol on experimental neuroses in cats. Psychosomatic Medicine, 1946, 8, 36-52.

Miller, N. E. Experimental studies of conflict. In J. McV. Hunt (Ed.), Personality and the behavior disorders. New York: Ronald Press, 1944.

Miller, N. E. Liberalization of basic S-R concepts: Extensions to conflict behavior, motivation and social learning. In S. Koch (Ed.), Psychology: A study of a science (Vol. 2). New York: McGraw-Hill, 1959.

Miller, N. E., \& BARRY, H. Motivational effects of drugs: Methods which illustrate some general problems in psychopharmacology. Psychopharmacologia, 1960, 1, 169-199.

(Received for publication August 9, 1976; revision accepted December 30, 1976.) 